\title{
Refractory NK-Cell Lymphoma, Unclassifiable
}

National Cancer Institute

\section{Source}

National Cancer Institute. Refractory NK-Cell Lymphoma, Unclassifiable. NCI Thesaurus.

Code C157690.

NK-cell lymphoma, unclassifiable that is resistant to treatment. 